This item was submitted to Loughborough's Research Repository by the author.

Items in Figshare are protected by copyright, with all rights reserved, unless otherwise indicated.

\title{
Mathematical question spaces
}

PLEASE CITE THE PUBLISHED VERSION

PUBLISHER

(c) Loughborough University

VERSION

AM (Accepted Manuscript)

LICENCE

CC BY-NC-ND 4.0

REPOSITORY RECORD

Sangwin, Christopher J.. 2019. "Mathematical Question Spaces”. figshare. https://hdl.handle.net/2134/4418. 
This item was submitted to Loughborough's Institutional Repository (https://dspace.lboro.ac.uk/) by the author and is made available under the following Creative Commons Licence conditions.

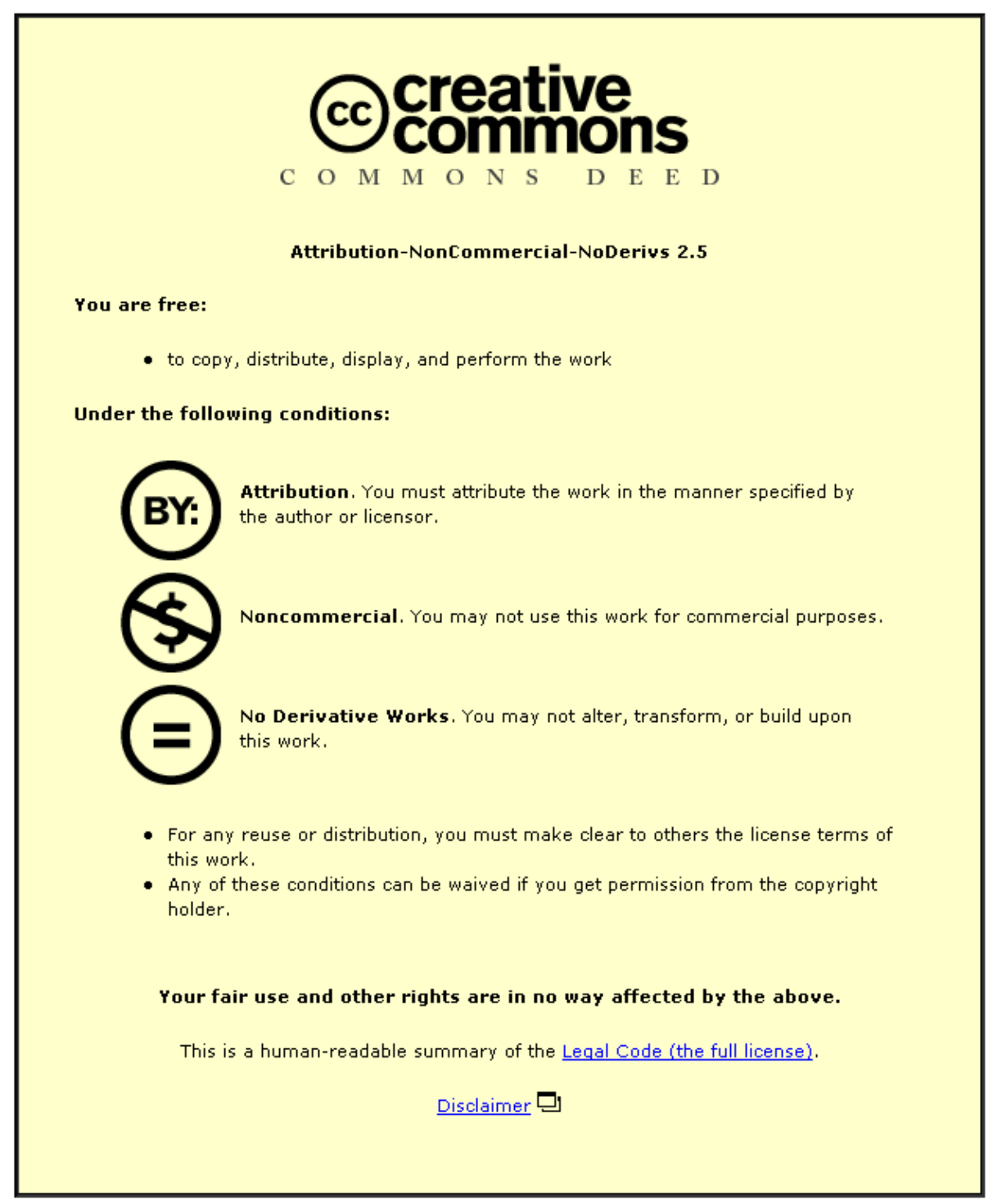

For the full text of this licence, please go to: http://creativecommons.org/licenses/by-nc-nd/2.5/ 


\section{MATHEMATICAL QUESTION SPACES}

Christopher J Sangwin 



\title{
Mathematical Question Spaces
}

\author{
Christopher J Sangwin \\ Maths Stats and OR Network \\ School of Mathematics \\ University of Birmingham \\ Birmingham \\ B152TT \\ C.J.Sangwin@bham.ac.uk
}

\section{Introduction}

It is uncontroversial to assert that learning mathematics is only effective when it is an active process on the part of the learner. Setting questions is a ubiquitous technique to engage students, and answering such questions constitutes a large proportion of the activity they undertake. Indeed, asking students questions is a central part of all theories of learning.

This paper examines in detail the process of randomly generating versions of mathematical questions for CAA. In doing this we examine not only a single mathematical question, but how such questions are linked together into coherent structured schemes. Two important pragmatic reasons are often cited by colleagues for wishing to generate a random sequence of questions.

- Randomly generated questions may reduce plagiarism

- Distinct but equivalent questions may be used for practice

Even if giving each student a distinct problem sequence reduces plagiarism, professional experience unfortunately demonstrates it is not eliminated. However, some students are well aware of the potential benefits of collaborative learning, possibilities for which are traditionally hard to provide in the mathematics classroom. As one student commented in their feedback evaluations:

"The questions are of the same style and want the same things but they are subtly different which means you can talk to a friend about a certain question but they cannot do it for you. You have to work it all out for yourself which is good."

Notice here the student voices the opinion that the questions "want the same things but they are subtly different". In this paper we address exactly this issue, by examining equivalent mathematical problems in some detail. 


\section{Mathematical Questions}

Linguistically, a question is a sentence worded or expressed so as to elicit information. We shall use the term "question" in such a way, when in practice many words are used in text books, for example "exercise", "problem", "task" and even "examples". Here, a question is also taken to include an instruction, such as "solve", "factor", "sketch" and so on.

Using schemes of questions is one of the major techniques used for selfstudy, home work or in the classroom. Working through such pre-structured exercises is akin to taking part in a dialogue, and such dialogues are an important part of learning. Although it is usual for a dialogue to take place between two interlocutors, an internal conversation occurs when one engages in "thinking aloud". On the nature of this internal conversation [7] says, "the mere act of communicating our ideas seems to help clarify them, for, in so doing, we have to attach them to words (or other symbols), which makes them more conscious". Hence, while one does not have a conversation with the textbook, the textbook may provoke internal enquiry and dialogue. They may also play a part in the learning process by providing mutual ground, or shared sequences of experiences, about which subsequent conversations can take place. There may be other legitimate uses, such as providing "finger exercises" to promote rather mindless, but nevertheless important, mechanical fluency.

A crucial distinction, when considering a mathematical question, is whether or not one cares about the answer. With many questions, no one cares about the actual answer. The purpose of the question is either to (i) practise some technique, or (ii) help build or reinforce some concept by prompting reflective activity. In other cases the purpose of the question is to obtain the answer. The question itself is a prototype of a practical problem which may be encountered, and hence this result may be useful.

We begin our examination of mathematical questions with a sequence of simple questions from [9]. This small, unassuming volume consists of 178 pages. There is no text or worked examples, instead simply sequences of problems. "These examples are intended to provide a complete course of elementary algebra for classes in which the bookwork is supplied by the teacher". Part of one such sequence is shown in Figure 1.

Draw the graphs of:

(1) $y=x^{2}$.

(2) $y=-x^{2}$.

(3) $y=2 x^{2}$.

(4) $y=x^{2}+2 \cdot 5$.

(5) $y=(x-1)^{2}$.

(6) $y=(x+2)^{2}+1$

(7) $y=x^{2}+4 x+6$.

(8) $y=x^{2}-3 x+1$

(9) Write out a general statement of the difference between the graphs of $y=x^{2}$ and of $y= \pm a\left\{(x-b)^{2}+c\right\}$.

Figure 1: [9], pg 62 
We claim that this sequence of questions is highly structured, and this example has been included here because clues to this structure are revealed in the unusual final synoptic question. Further that the purpose of such a sequence of questions is to develop concepts rather than obtain an answer or practice technique. Note however that including such a question may make little sense for a student who has struggled with questions (1)-(8), and has little work of merit from which to form a coherent synopsis. Question 1 provides a base from which comparisons can be made. Questions 2 and 3 rescale the y-axis. Question 4 is a vertical shift, question 5 is a horizontal one, and question 6 involves both. Questions 7 and 8 also require simple shifts, although some simple algebra is required to reveal precisely what these are.

Many books contain word problems where part of the process is setting up the equations themselves. This is modelling, in its broadest sense. Some of these problems are practical, others mathematical. What they have in common, is that the answer appears to be applied, and hence it is the answer which is important. They do not appear to be conceptual, nor for practice. Rather they might be termed utilitarian. The following (admittedly somewhat dated) example is taken from [1]. However similar (if not identical) examples may be found in many modern books.

Examples XXVII. b. 10. If 6 fewer bottles of wine can be bought for $£ 5$ when the price is raised ten shillings per dozen, what is the original price?

In many cases such exercises are highly structured, with examples carefully chosen to reveal different cases in the underlying mathematics.

A third category of questions are those which seek to practice some skill. For example, [1] Chapter XIV contains some 325 repetitive exercises on the topic of factoring quadratics alone. This large quantity of repetitive practice is typical of many algebra books, including modern ones. These sequences of problems tend to be highly structured. This structure includes things which are common to whole sequences of problems, for example integer roots, the signs of the roots are all positive, and things which are varied.

As a concrete example of constrained variation consider the following question.

$$
\text { Solve } a x^{2}+b x+c=0 \text {. }
$$

We might consider indexing the individual instances by using coordinates $(a, b, c)$. Clearly, there are some subspaces, such as the subspaces of mathematically possible questions. The subspace satisfying b2 ${ }^{3} 4 a c$ characterizes the question subspace with real solutions. While such a mechanical indexing of questions is technically feasible, we would like to consider a quite different issue. This is to draw an analogy with the concept of an example space developed by [10]. An example space is taken to be the cognitive domain possessed by the student, rather than some intrinsic mathematical space. We seek to develop a dual notion: that of mathematical question space. Just as with example spaces, the notions of the dimensions 
of possible variation and ranges of permissible change in any question space appear to be very useful. Each dimension of possible variation corresponds to an aspect of the question which can be varied to generate a collection different question instances. The range of permissible change is more problematic. "Permissible" may of course be taken to indicate the strict mathematical criteria of well-posedness, or may be used in a pedagogic sense. Given our educational context, a question space is considered to be the collection of instances which are educationally equivalent. That is to say, two instances in a space differ in ways which do not alter the purpose or effect of a question within that particular scheme. Furthermore, we identify the mathematical question with this pedagogic question space. While the student is likely to be aware only of the task in hand: the question instance, to the teacher this instance actually represents the question space and hence the underlying generality.

Clearly, the question space is more complex than simply varying a coefficient in a term. For example, in question 7 of the problem set shown in Figure 1, the question is an instance of a quadratic with no real roots, for which the completed square form is tractable. An instance of such a question would probably be given as an expansion of $(x-a)^{2}+b$, where $a$ is a small integer, and $b>0$ is a small integer. Hence, a particular dimension of variation certainly does not correspond to the direct variation of a coefficient in a question instance. As a result, to implement randomly generated instances from a question space sophisticated tools are necessary.

Clearly here it is easy to identify how the dimensions of variation affect the question instances, but it is unlikely that such an algebraic clarity will be evident in many situations. Equally, there is nothing to suppose that a dimension of variation will be algebraic at all. Variation could include which variable is used, the dimensions and orientation of geometric shapes, or the adjectives used in a word problem. Furthermore, there are many situations when a parameter will remain within a question, perhaps to suggest to the student that there is a range of permissible and "essentially the same" examples encapsulated within one question. It is possible in some circumstances that a question space will only contain one instance. For example, in Figure 1, question (1), there may be no reasonable alternatives, and the question space consists only of the instance "Draw the graph of $x^{2 n}$.

While practice of some technique could be seen to be the repeated completion of question instances from a particular question space, we argue that it is not. A selection of questions usually shows progression through a sequence of slightly different cases. Each of these will be consciously different, and so will be instances from different question spaces. 


\section{Existing Standards for CAA}

In this section we consider the data model for the representation of questions for CAA provided by the IMS Question \& Test Interoperability (QTI) specification. For them, an item is the smallest self contained exchangeable assessment object.

"An item is more than a 'Question' in that it contains the question and instructions to be presented, the response processing to be applied to the candidates response(s) and the Feedback that may be presented (including hints and solutions)."

Such a concept of a self contained item is present in virtually all CAA systems, either at an explicit or implicit level. In their sense it is significantly more than a question, since it contains details of response processing instructions, and feedback, both hints and solutions, to be given. This specification includes the notion of Item Clone, which are equivalent items created from an Item Template by the substitution of Item Variables. However, the specification operates only at the level of individual items, and takes no account of the sequence of items.

Similarly, the IMS Simple Sequencing Specification provides a mechanism for representing the intended behaviour of a "learning experience", the prototype of which is interactions with a sequence of items.

We argue that for mathematics the split between "item" and "sequence" is artificial and fails to capture crucially important aspects of the learning process in automated assessments built upon it. While it will be necessary to author and store items at this level, there is no clear distinction at the pedagogic level between item and sequence and it is often actually difficult to decide what the smallest exchangeable object is. Is a multi-part item a collection of separate items? While mathematics assessment can be shoe-horned into this data representation model, the results are unsatisfactory.

\section{The STACK CAA System}

This section concerns the implementation of a computer aided assessment (CAA) system for mathematics known as STACK: a System for Teaching and Assessment using a Computer algebra Kernel. A demonstration server is available at (http://www.stack.bham.ac.uk). As the names implies, STACK relies on a computer algebra system (CAS) at its heart to support a variety of tasks. The most important feature is that the CAA system evaluates the student answers containing mathematical content, rather than allow selection from a list of teacher provided answers, such as in multiple choice or multiple response questions.

Systems under which the processing of student answers is supported by computer algebra have gradually gained ground in higher education over the last five years. Perhaps the first system to make CAS a central feature was 
the AiM system, described by [2], with subsequent technical developments described in [8]. This system operates using Maple, as does the Wallis system of [3]. Other systems have access to a different CAS, such as CalMath which uses Mathematica, CABLE, see [4], which uses Axiom and the STACK system which uses the CAS Maxima. From private correspondence, the authors are also aware of systems which use Derive in a similar way.

Details of the question authoring process are given in [6], and the important issue of student input syntax in [5]. From our point of view we are most interested in random generation of structured mathematics questions. Experience with STACK and similar CAA systems demonstrated that virtually all necessary tasks can be performed with the following three functions, when backed up by the sophisticated library of CAS functions.

- Generate a random integer between 0 and $\mathrm{n}$.

- Generate a random floating point number between 0 and $\mathrm{n}$.

- Select a random item from a list.

The important issue is the availability of CAS, or CAS-like, functions which can be used to build structured mathematical objects. Describing this at a level of detail suitable for interpretability is a difficult task, and one unlikely to be completed in the near future.

STACK, as with the vast majority of contemporary CAA systems, currently only operates at the level of individual items. While it is clear how richer multipart items can be developed, it is not clear how technically separate but pedagogically connected items can be linked, to aid exchange and efficient re-use. This is the subject of ongoing work. 


\section{References}

[1] H. S. Hall. A School Algebra. MacMillian, London, 11th printing edition, 1929. First published 1912.

[2] S. Klai, T. Kolokolnikov, and N. Van den Bergh. Using Maple and the web to grade mathematics tests. In Proceedings of the International Workshop on Advanced Learning Technologies, Palmerston North, New Zealand, 4-6 December, 2000.

[3] M. Mavrikis and A. Maciocia. Wallis: a web-based ILE for science and engineering students studying mathematics. In Workshop of Advanced Technology for Mathematics Education in the 11th International Conference on Artificial Intelligence in Education, pages 505-512, Sydney, Australia, 2003.

[4] L. Naismith and C. J. Sangwin. Computer algebra based assessment of mathematics online. In Proceedings of the 8th CAA Conference 2004, 6th and 7th July, The University of Loughborough, UK, 2004.

[5] P. Ramsden and C. J. Sangwin. A liberalised mathematical syntax for computer-aided assessment. In Proceedings of the International Mathematica Symposium, Perth, Australia, 2005.

[6] C. J. Sangwin and M. J. Grove. STACK: addressing the needs of the "neglected learners". In Proceedings of the WebAlt Conference, Eindhoven, 2006.

[7] R. R. Skemp. The psychology of learning mathematics. Penguin, 1971.

[8] N. Strickland. Alice interactive mathematics. MSOR Connections, 2(1):2730, 2002. http://tsn.mathstore.ac.uk/newsletter/feb2002/pdf/aim.pdf (viewed December 2002).

[9] C. O. Tuckey. Examples in Algebra. Bell \& Sons, London, 1904.

[10] A. Watson and J. Mason. Extending example space as a learning/teaching strategy in mathematics. In A. D. Cockburn and E. Nardi, editors, Proceedings of the Annual Conference of the International Group for the Psychology of Mathematics Education (PME26, Norwich, United Kingdom), volume 4, pages 378-385, 2002. 